This is an author-created version of an article published in Beneficial Microbes. The original publication is available at:

https://www.wageningenacademic.com/doi/10.3920/BM2019.0197?url ver=Z39. 88-2003\&rfr id=ori:rid:crossref.org\&rfr dat=cr pub\%20\%200pubmed

Full citation reference:

Valent, D., L. Arroyo, E. Fàbrega, M. Font-i-Furnols, M. Rodríguez-Palmero, J.A. Moreno-Muñoz, J. Tibau, and A. Bassols. 2020. "Effects Of A High-Fat-Diet Supplemented With Probiotics And $\Omega 3$-Fatty Acids On Appetite Regulatory Neuropeptides And Neurotransmitters In A Pig Model". Beneficial Microbes 11 (4): 347-359. https://doi.org/10.3920/BM2019.0197

Document downloaded from: 


\title{
Effects of a high-fat-diet supplemented with probiotics and $\omega 3$-fatty acids on appetite regulatory neuropeptides and neurotransmitters in a pig model
}

\author{
D. Valent ${ }^{1}$, L. Arroyo ${ }^{1}$, E. Fàbrega ${ }^{2}$, M. Font-i-Furnols ${ }^{3}$, M. Rodríguez-Palmero ${ }^{4}$, J.A. \\ Moreno-Muñoz ${ }^{4}$, J. Tibau ${ }^{3}$, A. Bassols ${ }^{1, *}$ \\ ${ }^{1}$ Departament de Bioquímica i Biologia Molecular, Facultat de Veterinària. Universitat \\ Autònoma de Barcelona. Spain \\ ${ }^{2}$ Food Science-Institut de Recerca i Tecnologia Agroalimentàries, IRTA-Monells. Spain \\ ${ }^{3}$ Animal Science -Institut de Recerca i Tecnologia Agroalimentàries, IRTA- Monells. Spain \\ ${ }^{4}$ Laboratorios Ordesa S.L., Barcelona Science Park. Spain
}

*Corresponding author: anna.bassols@uab.cat

Running header: HFD, probiotics and $\omega 3$-fatty acids effects on food intake regulatory signals

\begin{abstract}
The pig is a valuable animal model to study obesity in humans due to the physiological similarity between humans and pigs in terms of digestive and associated metabolic processes. The dietary use of vegetal protein, probiotics and omega- 3 fatty acids is recommended to control weight gain and to fight obesity-associated metabolic disorders. Likewise, there are recent reports on their beneficial effects on brain functions. The hypothalamus is the central part of the brain that regulates food intake by means of the production of food intakeregulatory hypothalamic neuropeptides, as Neuropeptide Y (NPY), Orexin A and proopiomelanocortin (POMC), and neurotransmitters such as dopamine and serotonin. Other mesolimbic areas such as the hippocampus are also involved in the control of food intake. In this study, the effect of a high fat diet (HFD) alone or supplemented with these additives on brain neuropeptides and neurotransmitters was assessed in forty-three young pigs feed for 10 weeks with a control diet (T1), a high fat diet (HFD, T2), and HFD with vegetal protein supplemented with Bifidobacterium breve CECT8242 alone (T3) or in combination with omega-3 fatty acids (T4). A HFD provoked changes in regulatory neuropeptides and 3,4dihydroxyphenylacetic acid (DOPAC) in the hypothalamus and alterations mostly in the dopaminergic system in the ventral hippocampus. Supplementation of the HFD with B. breve CECT8242, especially in combination with long chain polyunsaturated omega-3 fatty acids, was able to partially reverse the effects of HFD. Correlations between productive and neurochemical parameters supported these findings. These results confirm that pigs are an appropiate animal model alternative to rodents for the study of the effects of HFD on weight gain and obesity. Furthermore, they indicate the potential benefits of probiotics and omega-3 fatty acids on brain function.
\end{abstract}

Keywords: Bifidobacterium, food-intake regulatory neuropeptides, hippocampus, hypothalamus, monoaminergic neurotransmitters 


\section{Introduction}

Obesity, which is characterized by excessive fat mass accumulation, has been a rising problem for the past several decades. Due to the importance of obesity and eating disorders, the investigation of food intake control mechanisms is a big issue (Stevens et al., 2012). This control is exerted by several physiological mechanisms, including stomach and gut-derived signals, and metabolic hormones such as leptin and insulin (Mantzoros et al., 2011). Besides these peripheral circuits, the regulation of appetite consists in a complex interaction between the body and the brain, which controls the homeostasis as well as the motivational (hedonic) aspects of food intake (Yu and Kim, 2012). In this context, one key interface linking the brain to the organism is the gut-brain axis, i.e., the multimodal bidirectional communication system between the gastrointestinal (GI) tract and the brain (Weltens et al., 2018).

The hypothalamus (HPT) is the central part of the brain that regulates food intake, coordinating global energy metabolism and appetite. It operates as a neuroendocrine transducer, sensing peripheral endocrine signals and transforming them into neurotransmitter (NT) events that deliver the homeostatic response back to the periphery (Yu and Kim, 2012). After a meal, circulating leptin enters the brain and reaches the arcuate nucleus (ARC), which is the main feeding behaviour-regulatory area in the HPT. In the ARC, two first-order neuron populations exist. One of them is a group of neurons producing the orexigenic peptides Neuropeptide Y (NPY) and Agouti-related peptide (AgRP) and the other is a subset of neurons expressing anorexigenic neuropeptides, like proopiomelanocortin (POMC) and cocaine- and amphetamine-regulated transcript (CART) (Schwartz et al., 2000; Sobrino Crespo et al., 2014). Leptin binding to its receptor in the ARC reciprocally activates the anorexigenic POMC/CART neurons and inhibits the orexigenic NPY/AgRP neurons leading to a decrease in food intake. Like leptin, insulin binds insulin receptors on ARC neurons, resulting in activation of POMC neurons and inhibition of NPY/AgRP neurons. These neuropeptides act on second-order neurons, localized in other hypothalamic areas. $\alpha$ Melanocyte stimulating hormone ( $\alpha$-MSH), produced by the posttranscriptional processing of POMC, activates catabolic pathways reducing food intake, thus acting as an anorexigenic neuropeptide, whereas the orexigenic neuropeptide Orexin A is produced in response to NPY/AgRP (Fernø et al., 2015). High fat diet induced-obesity is also characterized by alterations in the HPT neuropeptide pattern, although some controversy exists in the literature due to the diversity of animal models, diet composition and duration of these studies (Gumbs et al., 2016).

Although it is clear that the HPT has a main role in the control of the homeostasis of body weight, it is becoming evident that a relationship exists between the hypothalamic circuits and the mesolimbic and mesocortical monoaminergic systems (Gumbs et al., 2016). For instance, orexin neurons project onto dopaminergic neurons in the ventral tegmental area (VTA) and connect orexigenic signals to the mesocorticolimbic dopamine (DA) system of the brain (van Zessen et al., 2012). DA is believed to be mainly anorexigenic since leptin acts on DA neurons in the VTA to suppress feeding (Hommel et al., 2006). On the other hand, serotonin (5-HT) acts on POMC neurons through the 5HT-2C receptor to induce anorexia (Lam et al., 2010). Outside the HPT, several authors have highlighted the deleterious effects of a HFD on the hippocampus (HC) function, mostly related to cognition, including memory processes, cognitive functions, learning capacities and motor abilities (Beck and Pourié, 2013; Krishna et al., 2015). 
The concept of the gut-brain axis has been recently enriched with the evidence pointing to the influence of the gut microbiota being essential not only for gut health but also for normal physiologic functioning in other organs, especially the brain, giving rise to the "brain-gutmicrobiome axis", defined as the bidirectional communication system enabling gut microbes to communicate with the brain and the brain with the gut (Bienenstock et al., 2015; Dinan and Cryan, 2017). The use of vegetal protein and probiotics have been largely suggested as therapeutic strategies to fight HFD-induced obesity as well as obesity-associated pathologies (Arora et al., 2013; Nova et al., 2016). Several species and strains of Lactobacillus and Bifidobacterium have been used in rodent animal models that have demonstrated positive effects on obesity reduction (Chen et al., 2012; Kondo et al., 2010; Shin et al., 2017), obesityassociated inflammation (Cano et al., 2013), lipid metabolism (Moya-Pérez et al., 2014; Yoo et al., 2013), metabolic syndrome (Kondo et al., 2013), insulin-sensitivity (Bagarolli et al., 2017), HFD-altered vascular function (Mauricio et al., 2017), HFD-induced colitis (Lim and Kim, 2017) and steatohepatitis (Reichold et al., 2014). Studies in human patients have also reported the beneficial effects of probiotics on metabolic syndrome and obesity (Bernini et al., 2016; Minami et al., 2015). Similarly, dietary omega-3 fatty acids improve obesity-associated metabolic disorders in mice and humans (Huang et al., 2016; Lorente-Cebrián et al., 2013; Martínez-Fernández et al., 2015). Although more scarce, there are recent reports on the beneficial effects of probiotics and unsaturated fatty acids on cognitive functions in human studies and rodent models (Beilharz et al., 2015; Kang and Gleason, 2013; Noble et al., 2017). In the scope of the gut-brain-microbiome axis, the name of psychobiotics is starting to be used in the literature, related to the recent research field that relates the microbiota of the intestine with diseases of the nervous system and its possible treatment (Bermúdez-Humarán et al., 2019).

The majority of studies on the brain components of appetite control have been performed in rodents, but these animal species present some limitations when compared to humans, in metabolic as well as neuronal aspects. Instead, pigs have been used as a model for obesity and obesity-related diseases due to the physiological similarity between humans and pigs in terms of digestive and associated metabolic processes (Bassols et al., 2014; Koopmans and Schuurman, 2015). The interaction with gut microbiota has been also studied in the porcine model (Heinritz et al., 2013). Furthermore, in an ongoing study in a porcine model fed with a HFD, we have previously showed that the use of rice protein hydrolysate, the probiotic Bifidobacterium breve CECT8242 and omega-3 fatty acids prevented excessive weight gain and dyslipidemic alterations associated with obesity in this model (Serrano et al., 2016).

The objective of the present work was to analyse the effect of a HFD on brain monoaminergic neurotransmitters (NTs) and neuropeptides involved in the regulation of appetite in young pigs. Under the hypothesis of the gut microbiota - brain axis, the second goal was to ascertain whether, in animals fed this HFD, the use of a rice protein hydrolysate, with $B$. breve supplementation alone or in combination with omega-3 fatty acid was able to counterbalance these central effects. In the overall, we wanted to assess whether changes in food intake or body weight induced by these dietary supplements could be explained by a modification of the food intake-regulatory neuropeptides and NTs.

\section{Experimental section}

\section{Study design}

Animals used in this experiment (female, $\mathrm{n}=43$ ) were obtained from 12 swine pure breed Duroc contemporary litters. After weaning, animals were moved to the IRTA experimental 
station starting a period of adaption feed with a standard diet. Care and management procedures were approved by the IRTA bioethics committee (DAAM 7306/2013). At 9 weeks of age animals were located in environmentally monitored facilities, randomly distributed in 4 pens (10 to 11 animals per pen from different litters) and fed ad-libitum for 10 weeks with 4 different diets (treatments T1 to T4). Each pen had a partly slatted floor (60\% solid concrete and $40 \%$ slatted), with some sawdust provided on the concrete floor on a regular basis and a natural light cycle, with a minimum of intensity of 40 lux (EU legislation on pig welfare) and 8 hours light. The room temperature was maintained at $22+-5^{\circ} \mathrm{C}$.

Fresh water was provided to all animals and individual daily voluntary feed consumption was obtained using an automatic feeding recording system. Weight gain and food intake were obtained weekly during the growing period. Experimental diets were elaborated at the IRTA experimental mill, with different components: cereal, vegetal or milk protein, starch, sugar and minerals, micronutrients and amino acids in different percentages (see Table S1).

Estimated nutritive composition of diets are reported in Table 1. All animals received an additional daily ration of 18 grams soft cheese (with a $14 \%$ crude protein and $14 \%$ fat with a total of $42 \mathrm{Kcal}$ of energy). T1 was a control diet according to the NRC (Nutrition Resource Center) recommendations. T2 was a western diet formulated with a high fat content of animal origin (lard) and protein of animal origin (caseinate). T3 and T4 contained soft cheese including $5 \cdot 10^{10} \mathrm{cfu} /$ day $B$. breve CECT 8242 probiotic. T4 included long chain polyunsaturated omega-3 fatty acids (1 $\mathrm{g}$ stearidonic acid (SDA) and $2 \mathrm{~g}$ docosahexaenoic acid (DHA) per $100 \mathrm{~g}$ fat).

Supplementary Table S1. Components of experimental diets T1 to T4 (in percentage)

Table 1. Nutritive composition of experimental diets (percentages versus total food weight)

Treatment

\begin{tabular}{lcccc}
\hline & $\mathrm{T} 1$ & $\mathrm{~T} 2$ & $\mathrm{~T} 3$ & $\mathrm{~T} 4$ \\
\hline Crude Protein (\%) & 17,6 & 14,34 & 14,54 & 14,62 \\
Crude Fibre (\%) & 11 & 1,71 & 1,75 & 1,74 \\
Fat (\%) & 4,6 & 11,55 & 11,92 & 12,09 \\
Sodium \% & 0,15 & 0,15 & 0,15 & 0,15 \\
Calcium \% & 0.80 & 0.70 & 0.80 & 0.80 \\
Ash (\%) & 5.55 & 4.13 & 4.15 & 4.15 \\
Lysine (\%) & 1,03 & 0,93 & 0,93 & 0,93 \\
Phosphorous (\%) & 0.56 & 0.49 & 0.51 & 0.51 \\
Lysine (\%) & 0.99 & 0.90 & 0.90 & 0.90 \\
Threonine (\%) & 0.66 & 0.57 & 0.57 & 0.57
\end{tabular}




$\begin{array}{lcccc}\text { Methionine (\%) } & 0.36 & 0.37 & 0.37 & 0.37 \\ \text { Tryptophan (\%) } & 0.23 & 0.20 & 0.18 & 0.18 \\ \text { Energy (MJ/kg) } & 10,83 & 15,16 & 15,56 & 15,56 \\ \begin{array}{l}\text { Energy (kcal/kg) } \\ \text { Bifidobacterium breve }\end{array} & 2587 & 3621 & 3716 & 3716 \\ \text { CECT8242 (cfu/day) } & - & - & 5 \cdot 10^{10} & 5 \cdot 10^{10} \\ \begin{array}{l}\text { Omega-3 fatty acids (\% } \\ \text { total fatty acids) }\end{array} & - & - & - & 3 \\ \end{array}$

\section{Brain collection and dissection}

Animals were fasted $8 \mathrm{~h}$ before being transported from the experimental farm to the experimental slaughterhouse (1.2 km of distance). After the unloading, pigs were located in the lairage pens for an hour. Animals were stunned in groups of two by exposure to $90 \% \mathrm{CO}_{2}$ at atmospheric air for 3 min and exsanguinated afterwards.

Brain was removed from skull immediately after slaughter. Brain areas (prefrontal cortex (PFC), amygdala (Amg), ventral hippocampus (VHC), dorsal hippocampus and hypothalamus (HPT)) were excised as quickly as possible, frozen in liquid nitrogen and kept at $-80{ }^{\circ} \mathrm{C}$ until analysis.

\section{Determination of neurotransmitters by HPLC}

Brain samples were weighted and homogenized by sonication (Branson Digital Sonifier 250, Branson Ultrasonics Corp., Danbury, CT) with $0.3 \mathrm{mg}$ tissue/ $1 \mu \mathrm{L}$ lysis buffer (150 $\mathrm{mMNaCl}, 50 \mathrm{mMTris}-\mathrm{HCl}, 0.1 \% \mathrm{NP}-40$ and $\mathrm{pH}$ 7.5). This lysis buffer is compatible with all techniques used in this study. Dihydroxybenzylamine (DHBA) was added to the lysis buffer at $300 \mathrm{pg} / \mu \mathrm{L}$ as internal standard. Proteins in brain lysates were precipitated by adding 0.25 $\mathrm{M}$ perchloric acid containing $0.1 \mathrm{M}$ sodium metabisulfite $\left(\mathrm{Na}_{2} \mathrm{~S}_{2} \mathrm{O}_{5}\right)$ and $0.25 \mathrm{M}$ ethylenediaminetetraacetate (EDTA)) in a $1.5(\mathrm{v} / \mathrm{v})$ ratio. Finally, samples were centrifuged at $12000 \mathrm{~g}$ for 10 minutes at $4{ }^{\circ} \mathrm{C}$ and the supernatant was kept at $-80{ }^{\circ} \mathrm{C}$ until analysis.

The concentration of NTs from different brain areas was determined by high performance liquid chromatography (HPLC) in an Elite LaChrom system (Merck-Hitachi, Prague, The Czech Republic) equipped with Cromolith Rp-18e column (Merck, Darmstadt, Germany) with electrochemical detection (ESA Coulochem II 5200). The mobile phase consisted of 500 $\mathrm{mM}$ citrate buffer $\mathrm{pH}$ 2.8-3.0, 0.05 mM EDTA, $1.2 \mathrm{mM}$ sodium octyl sulphate (SOS) and $1 \%$ acetonitrile. The flow rate was $1 \mathrm{~mL} / \mathrm{min}$ and the voltage applied to the detector was set at 400 $\mathrm{mV}$. Twenty $\mu \mathrm{L}$ of supernatant were injected in the HPLC for the quantification of noradrenaline (NA), dopamine (DA) and its metabolites 3,4-dihydroxyphenylacetic acid (DOPAC) and homovanillic acid (HVA) and the indoleamines serotonin (5-HT) and its metabolite 5-hydroxyindoleacetic acid (5-HIAA) (Arroyo et al., 2016).

\section{Quantification of neuropeptides}

Neuropeptides were determined in lysates prepared from hypothalamus. The orexigenic neuropeptides Orexin A (OrexA) and Neuropeptide Y (NPY) and the anorexigenic neuropeptide Proopiomelanocortin (POMC) were quantified by ELISA (EIA anti-Orexin A, Phoenix Pharmaceuticals, Burlingame, CA, USA; EIA Porcine Anti-Neuropeptide Y, Phoenix 
Pharmaceuticals, Burlingame, CA, USA; ELISA Porcine Anti-POMC, Neobiolab, Cambridge, MA, USA).

\section{Statistical analysis}

Data presented on this study was analysed using IBM SPSS software (IBM SPSS v22). Shapiro-Wilk normality test of descriptive data was performed and logarithmic data was transformation of data was calculated if necessary. ANOVA and Tukey as a Post-Hoc test was applied, with a $\mathrm{P}<0.05$ of significance level and $0.05 \leq \mathrm{P} \leq 0.1$ for tendency, to analyse differences between conditions. Correlation between variables was analysed with two-tailed Pearson's test. Only correlations with coefficients higher than 0.5 and $\mathrm{P}<0.05$ were considered for analysis.

\section{Results}

\section{Productive results}

Data on weight, weight gain and food intake are shown in Table 2. All four groups had similar initial weight at week 9. At week 18, at the end of diet treatments, animals in T3 and T4 had similar weight than those in T1 (control diet) and lower than T2 (high-fat diet, HFD) $(P=0.02)$. A similar result is observed when measuring the weight gain throughout the experiment (week 9 to week 18) $(P==0.01)$ and the ADG $(P=0.01)$. In all cases, there were no differences between T1, T3 and T4.

Regarding food intake measured in $\mathrm{kg}$, there were no differences due to HFD vs the control, in total food intake or daily food intake. Nevertheless, treatment with probiotics and omega-3 fatty acids (T4) decreased food intake when compared to the control group ( $P=0.002)$. A tendency was observed when T3 and T4 were compared to T2 $(P=0.057)$.

When determining food intake measured in kcal, individuals in the HFD group increased their total and daily energy intake when compared to the control group. Animals in T3 and T4 also had higher energy intake when compared to T1 $(P<0.001)$. A tendency was observed for the T4 group when compared to T2 $(P=0.076)$.

Table 2. Effects of dietary treatments on weight gain, average daily gain (ADG) and food intake in young pigs

\begin{tabular}{lcccc}
\hline Treatment & T1 & T2 & T3 & T4 \\
\hline $\mathrm{n}$ & 11 & 10 & 11 & 11 \\
\hline & mean +/- SE & Mean +/- SE & Mean +/- SE & Mean +/- SE \\
\hline Weight W9 (kg) & $16,50+/-0,86$ & $16,70+/-0,70$ & $16,14+/-0,59$ & $16,27+/-0,41$ \\
Weight W18 (kg) & $53,27+/-1,74^{\mathrm{a}, \mathrm{b}}$ & $60,00+/-2,65^{\mathrm{b}}$ & $51,36+/-2,12^{\text {a }}$ & $50,23+/-2,07^{\text {a }}$ \\
Weight gain W9-W18 (kg) & $36,77+/-1,27^{\mathrm{a}, \mathrm{b}}$ & $43,30+/-2,26^{\mathrm{b}}$ & $35,23+/-1,62^{\mathrm{a}}$ & $33,95+/-1,90^{\text {a }}$ \\
ADG W9-W18 (kg) & $0,583+/-0,020^{\mathrm{a}, \mathrm{b}}$ & $0,687+/-0,036^{\mathrm{b}}$ & $0,559+/-0,026^{\mathrm{a}}$ & $0,539+/-0,030^{\mathrm{a}}$ \\
Food Intake W9-W18 (kg) & $92,12+/-1,72^{\mathrm{b}}$ & $89,48+/-2,80^{\mathrm{a}, \mathrm{b}}$ & $82,67+/-2,66^{\mathrm{a}, \mathrm{b}}$ & $79,57+/-3,34^{\mathrm{a}}$
\end{tabular}




\begin{tabular}{|c|c|c|c|c|}
\hline Daily Food Intake W9-W18 (kg) & $1,462+/-0,028^{b}$ & $1,419+/-0,443^{a, b}$ & $1,314+/-0,042^{a, b}$ & $1,264+/-0,053^{a}$ \\
\hline Food Intake W9-W18 (kcal) & $240,65+/-4,49^{a}$ & $328,48+/-10,30^{b}$ & $307,24+/-8,99^{b}$ & $295,70+/-12,39^{b}$ \\
\hline Daily Food Intake W9-W18 (kcal) & $3,82+/-0,07^{a}$ & $5,21+/-0,16^{\mathrm{b}}$ & $4,88+/-0,16^{\mathrm{b}}$ & $4,69+/-0,20^{b}$ \\
\hline
\end{tabular}

Different superscript indicates significant differences $(P<0.05)$

\section{Brain neurotransmitter profile}

NTs of the catecholamine and indoleamine pathways were analysed in HPT and HC, and results compared between treatments in each brain region. No significant changes were observed in the PFC and the amygdala (not shown). Most of the observed changes are related to the dopaminergic pathway (Table 3 ).

In the HPT, the concentration of DOPAC in groups treated with probiotics (T3 and T4) was similar to control and lower than T2 $(P=0.016)$. The HC was divided in two areas, the dorsal and ventral HC, due to their different functional role (Fanselow and Dong, 2010). No significant differences were observed in dorsal HC. In the ventral HC, a decrease in dopaminergic NTs was observed, the most relevant change being the decrease in DA in T2 compared to T1 $(P=0.065)$. A similar pattern was observed for the metabolite DOPAC, although it did not reach statistical significance $(P=0.072)$, which was partially reversed in T4. The total DA-system, calculated as the sum of dopaminergic compounds (DA, DOPAC and HVA) also decreased in T2 $(P=0.049)$ and was reversed by supplementation, especially in T4 $(P=0.023)$. Furthermore, a tendency to decrease in the serotoninergic metabolite 5HIAA was also observed in T2 vs T1, which was restored in T4 $(P=0.096)$.

Altogether, these results show that the brain system which is mainly affected by HFD is the dopaminergic pathway in the ventral $\mathrm{HC}$ of the brain regions measured. Diets with probiotics, especially when supplemented also with $\omega 3$-fatty acids, restore at least partially the alterations induced by HFD (Figure 1).

Table 3. Effects of dietary treatments on the concentration of monoaminergic neurotransmitters in pig brain areas (ng/g tissue)

\begin{tabular}{lcccc}
\hline Treatment & T1 & T2 & T3 & T4 \\
\hline $\mathrm{n}$ & 11 & 9 & 12 & 12 \\
\hline Hypothalamus & mean $+/$ - SE & mean + - SE & mean $+/$ - SE & mean $+/$ - SE \\
\hline NA & & & & \\
DOPAC & $2082.48 \pm 219.36$ & $2036.89 \pm 312.41$ & $1906.36 \pm 186.33$ & $1883.54 \pm 125.65$ \\
DA & $309.20 \pm 37.26^{\mathrm{a}, \mathrm{b}}$ & $343.14 \pm 28.19^{\mathrm{a}}$ & $226.71 \pm 25.74^{\mathrm{b}}$ & $227.33 \pm 23.37^{\mathrm{b}}$ \\
HVA & $228.38 \pm 26.10$ & $206.28 \pm 32.40$ & $200.98 \pm 16.87$ & $226.77 \pm 30.33$ \\
5-HT & $481.98 \pm 51.74$ & $537.64 \pm 71.53$ & $441.47 \pm 64.74$ & $492.23 \pm 81.28$ \\
5-HIAA & $1055.11 \pm 98.98$ & $1134.32 \pm 158.96$ & $1074.31 \pm 76.01$ & $1104.07 \pm 130.46$ \\
& $432.38 \pm 38.25$ & $437.35 \pm 54.99$ & $392.63 \pm 27.55$ & $449.93 \pm 42.51$
\end{tabular}




\section{Dorsal hippocampus}

\begin{tabular}{lcccc}
\hline NA & $308.76 \pm 10.13$ & $342.50 \pm 17.36$ & $351.31 \pm 17.67$ & $339.48 \pm 12.63$ \\
DOPAC & $147.56 \pm 20.40$ & $145.90 \pm 19.31$ & $186.64 \pm 31.40$ & $125.66 \pm 15.55$ \\
DA & $\mathrm{ND}$ & $\mathrm{ND}$ & $\mathrm{ND}$ & $\mathrm{ND}$ \\
HVA & $225.18 \pm 39.03$ & $188.13 \pm 10.71$ & $183.94 \pm 24.96$ & $215.31 \pm 19.19$ \\
5-HT & $\mathrm{ND}$ & $\mathrm{ND}$ & $\mathrm{ND}$ & $\mathrm{ND}$ \\
5-HIAA & $325.60 \pm 37.82$ & $291.14 \pm 32.50$ & $304.21 \pm 13.44$ & $309.10 \pm 16.98$ \\
\hline Ventral hippocampus & & & & \\
\hline NA & $279.58 \pm 11.44$ & $290.62 \pm 19.06$ & $283.73 \pm 6.11$ & $295.35 \pm 11.21$ \\
DOPAC & $211.87 \pm 24.90^{\mathrm{a}, \mathrm{b}}$ & $169.64 \pm 10.61^{a}$ & $207.68 \pm 14.05^{\mathrm{a}, \mathrm{b}}$ & $234.40 \pm 18.13^{b}$ \\
DA & $142.49 \pm 10.72^{a}$ & $91.98 \pm 8.96^{b}$ & $109.19 \pm 11.32^{\mathrm{a}, \mathrm{b}}$ & $122.40 \pm 18.78^{\mathrm{a}, \mathrm{b}}$ \\
HVA & $190.98 \pm 10.46^{a}$ & $167.21 \pm 11.40$ & $179.11 \pm 13.65$ & $200.47 \pm 16.15$ \\
Total DA system & $545.34 \pm 117.03^{\mathrm{a}}$ & $428.82 \pm 57.34^{\mathrm{b}}$ & $495.98 \pm 74.12^{\mathrm{a}, \mathrm{b}}$ & $557.26 \pm 128.77^{\mathrm{a}}$ \\
5-HT & $\mathrm{ND}$ & $\mathrm{ND}$ & $\mathrm{ND}$ & $\mathrm{ND}$ \\
5-HIAA & $330.65 \pm 26.96^{\mathrm{a}, \mathrm{b}}$ & $275.47 \pm 11.27^{a}$ & $297.93 \pm 14.06^{\mathrm{a}, \mathrm{b}}$ & $336.34 \pm 14.61^{b}$
\end{tabular}

ND: non detectable

Different superscript indicates significant differences $(P<0.05)$. Superscript in italics indicates statistical tendencies $(P<0.1)$

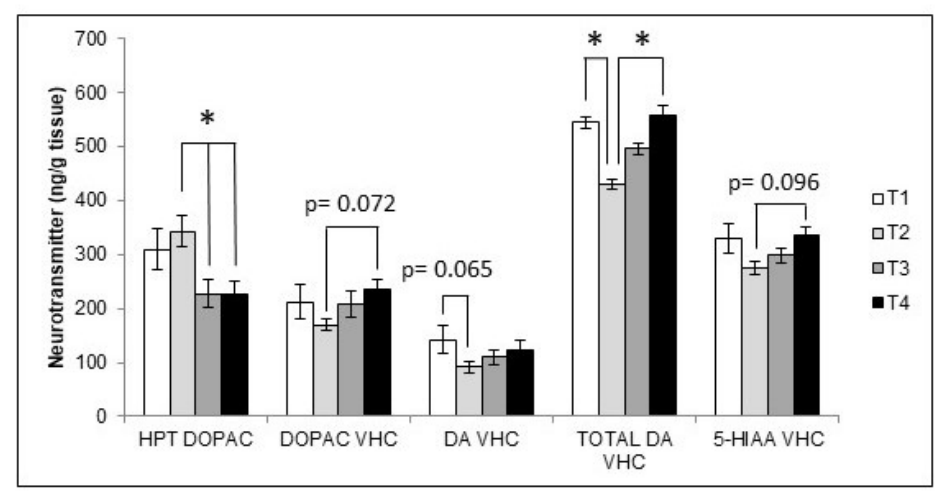

Figure 1. Alterations in neurotransmitter concentration exerted by dietary treatment in hypothalamus (HPT) and ventral hippocampus (VHC).

\section{Neuropeptide profile in the hypothalamus}

Results obtained by ELISA in hypothalamic extracts are shown in Figure 2 and Table 4 . The orexigenic neuropeptides Orexin A and NPY showed similar patterns since they were lower in HFD compared to the control group T1. The decrease was reversed in the probiotic-treated 
groups T3 and T4. In contrast, the concentration of the anorexigenic peptide POMC increased after HFD and was partially normalized when the animals were treated with probiotics with or without omega-3 fatty acids. Nevertheless, differences did not reach statistical significance possibly due to the high biological variability between individuals.

Table 4. Effects of dietary treatments on the concentration of neuropeptides in pig hypothalamus (ng/g tissue)

\begin{tabular}{lcccc}
\hline Treatment & T1 & T2 & T3 & T4 \\
\hline $\mathrm{n}$ & 11 & 9 & 12 & 12 \\
\hline Hypothalamus & mean + - SE & mean + - SE & mean + - SE & mean + - SE \\
\hline Orexin A & $2.15 \pm 0.96$ & $1.45 \pm 0.46$ & $2.38 \pm 0.70$ & $2.97 \pm 1.04$ \\
NPY & $50.24 \pm 11.89$ & $29.71 \pm 3.34$ & $40.49 \pm 5.37$ & $39.15 \pm 4.59$ \\
POMC & $9.57 \pm 2.45$ & $15.62 \pm 4.38$ & $12.44 \pm 1.87$ & $13.50 \pm 2.30$ \\
\hline
\end{tabular}

Figure 2. Hypothalamic neuropeptide concentration in young pigs under different dietary treatments.

\section{Correlation between variables}

All results from productive data and NT and neuropeptide data were analysed with Pearson correlation according to different brain areas and diets. Results are presented in Figure 3 and 4 as heatmaps from the same treatment and brain area. Only results for HPT and ventral HC are presented since those were the areas with significant results. Only correlations with coefficients higher than \pm 0.5 and $\mathrm{P}<0.05$ were considered for analysis.

1) Hypothalamus

In the control treatment T1, positive correlations were found between NTs in the dopaminergic pathway (DA, DOPAC and HVA), between DA and NA and between the serotoninergic pathway and the catecholamines. Neither significant positive nor negative correlations were found between productive parameters and NT or neuropeptides. When comparing T1 with T2 (HFD), clear differences were observed. First of all, the correlation between all productive parameters was tighter in T2 than in T1 with coefficients higher than 0.8 . Interestingly, a high correlation was found between productive parameters and 5-HT and its metabolite 5-HIAA and also between productive parameters and DOPAC and HVA, that were not observed in T1. A clear negative correlation was found between the orexigenic neuropeptide NPY and the NTs DA and 5-HT.

Groups supplemented with probiotics (T3 or T4) presented different correlation profiles than $\mathrm{T} 1$ or $\mathrm{T} 2$. The expected correlations in productive data and within NTs were also found. In T3, no other correlations were detected except a positive correlation between NA and the neuropeptides, specially Orexin A and POMC. In T4, a positive correlation was found between productive data and the orexigenic neuropeptides Orexin A and NPY, whereas a negative correlation was found between productive data, 5-HIAA and, in some cases, DOPAC or HVA. A negative correlation appeared also between Orexin A and DA and 5HIAA, and also between NPY and 5-HIAA. 
Figure 3. Correlation heatmaps between productive parameters, neuropeptides and NTs in the HPT of pigs under different dietary treatments. Light grey colour represents positive correlations and dark grey colour represents negative correlations. Numbers correspond to correlation coefficients.

\section{2) Ventral Hippocampus}

Heatmaps profiles in ventral HC were different than in HPT. High correlation coefficients between productive parameters were found in all treatments, which were tighter in T2, T3 and T4 than in T1.

Very few correlations were found between productive parameters and NTs. The exception was T1, where a negative correlation was observed between food intake and DOPAC and HVA (DA metabolites) and also to 5-HIAA as well as a positive correlation between productive parameters and NA. Besides, a positive correlation between productive parameters and HVA was observed in T2 and only a few correlations between NTs in T3 and T4. In all cases, correlation coefficients are lower than in the HPT.

Figure 4. Correlation heatmaps between productive parameters, neuropeptides and NTs in the VHC of pigs under different dietary treatments. Light grey colour represents positive correlations and dark grey colour represents negative correlations. Numbers correspond to correlation coefficients.

\section{Discussion}

In the pig model used in the present study, the effects of HFD (T2) compared to the control group (T1) were first evaluated. It should be taken into account that brain samples in these pigs were obtained in animals fasted for 8 hours. Indeed, plasma insulin concentration did not differ between groups (data not shown). This implies that the changes described in the present work are not due to the short-term response after a meal. This discussion will approach the changes provoked by HFD and their potential restoration after dietary intervention with probiotics and/or omega-3 fatty acids separately in the HPT and the ventral HC, since it is believed that the HPT is the brain area mainly responsible for the homeostatic control of food intake, whereas the $\mathrm{HC}$ is involved in the hedonic control, i.e., other aspects of appetiteinfluencing behaviours such as mood and reward.

\section{The effects of a HFD (T2 versus T1) in the NT and neuropeptide profiles in HPT and ventral $H C$}

In the HPT, the orexigenic NPY and Orexin A decreased whereas the anorexigenic peptide POMC increased, similar to many models of diet-induced obesity in rodents. Although the specific effects may depend on the duration and type of the dietary intervention, it has been generally described that HFD decreased NPY in the HPT, at the mRNA as well as peptide level, especially after a prolonged HFD, similar to the present porcine model (Dziedzic et al., 2007; Gumbs et al., 2016; Morris et al., 2008; Stricker-Krongrad et al., 1998; Wang et al., 2002). Similar results have been reported with OrexinA (Nobunaga et al., 2014; Park et al., 2004). In the obesity-susceptible C57BL/6J strain of mice subjected to HFD for 2 weeks, an increase in the expression of POMC has been also described (Ziotopoulou et al., 2000). In the present study, differences did not reach statistical significance possibly due to the high individual variability, but the data existing in the literature support our results. Furthermore, 
an increase in the concentration of DOPAC was observed, indicating an increase in the dopaminergic pathway activity. DA is considered to be an anorexigenic signal in the HPT, which is involved in the homeostatic control of food intake (Schwartz et al., 2000). Strong positive correlations between DOPAC or HVA and food intake or weight gain confirmed those relations between the dopaminergic pathway and food intake. Concurrently, a positive correlation was observed between 5-HT or 5-HIAA and food intake or weight gain, as described (Lam et al., 2010). The correlation heatmap obtained in T2 also reveals negative correlations between NPY and DA or 5-HT which support the hypothesis of DA and 5-HT having an anorexigenic role in the HPT (Lam et al., 2010; Schwartz et al., 2000), and also suggesting an inhibitory interaction between NPY and DA or 5-HT.

In the HC, the dopaminergic system is mostly affected by HFD, since there is a decrease in DA, DOPAC, as well as the sum of all DA metabolites (total DA system), indicating inactivation of this pathway. The decrease in the DA pathway was observed in the ventral $\mathrm{HC}$, which is believed to be related to emotion, stress and affect, whereas the role of dorsal $\mathrm{HC}$ is related to cognitive functions (Fanselow and Dong, 2010). As mentioned above, after food intake or a HFD, the DA decrease observed on ventral HC could be provoked by the inhibition of the mesocorticolimbic DA system in the VTA where the DA secretion is inhibited directly by leptin (Palmiter, 2007) and $\alpha-\mathrm{MSH}$ via intermediate GABAergic synapses between ARC and VTA (van Zessen et al., 2012). These results confirm the notion of the orexigenic function of DA in brain areas like the HC (Schwartz et al., 2000). The correlation heatmaps in the HC were quite different from the HPT. A negative correlation is observed between weight gain and food intake and the monoaminergic pathways.

The effects of a dietary intervention (T3 and T4) in the NT and neuropeptide profiles in HPT and ventral $H C$

The inclusion of $B$. breve CECT 8242 as probiotic alone (T3) or together with omega-3 fatty acids (T4) caused changes in brain neuropeptides and NTs that partially reversed the alterations caused by HFD. In short, ventral HC dopaminergic parameters increased when compared to HFD to become similar to the control diet. Furthermore, hypothalamic DOPAC which increased in HFD, decreased to lower values than in the control diet. When comparing the effects of T3 and T4, the overall results suggested that the concurrent addition of longchain derivatives of polyunsaturated essential fatty acids, such as DHA or SDA to the diet normalized up to a higher degree the concentrations of neuropeptides and NTs in the ventral HC and the HPT when added to a HFD.

Our results are in accordance to the recent indications that a microbiota gut-brain axis exists by which gut microorganisms modulate neuroendocrine and neurological mechanisms (Rhee et al., 2009). This fact has opened the possibility of modulating brain function and behaviour with probiotics, prebiotics and diet (Bermúdez-Humarán et al., 2019; Liu et al., 2016). For instance, changes in gut microbiota can modulate the peripheral and central nervous systems, resulting in altered brain functioning (Bienenstock et al., 2015; Mayer et al., 2015). Likewise, changes in gut microbiota may influence eating behaviour, memory, mood, and cognition and may be clinically relevant to a range of disorders, including anxiety or depression (Bauer et al., 2019; Galland, 2014; Liu et al., 2016) and eating disorders (van de Wouw et al., 2017). Even severe neurological disorders like autism, schizophrenia and neurodegenerative diseases have been linked to the gut microbiota (Bermúdez-Humarán et al., 2019; Westfall et al., 2017). Likewise, specific probiotic strains of Bifidobacteria have been described to exert stress-related changes in behaviour and physiology (Savignac et al., 2015, 2014), whereas probiotic supplementation may positively impact learning capacity and LTP in rats with Alzheimer disease, most likely via the release of NTs or via a protective effect on serum lipid 
profiles (Rezaeiasl et al., 2019). The use of several probiotics including B. breve has been reported as having antidepressive effects in mice and human patients (Yong et al., 2020). The evidences come also from studies directly linking the effects of probiotics to brain NTs or neuropeptides, mostly to dopaminergic neurotransmission (González-Arancibia et al., 2019). As examples, it has been reported that Lactobacillus strains may modulate neurochemicals related to affective disorders (DA, 5-HT) (Liu et al., 2016), that prebiotics may modulate anxiety through an effect on 5-HT receptors (Savignac et al., 2016), Clostridium butyricumbased probiotic may also modulate hypothalamic DA and 5-HT (Cao et al., 2019). In vitro, Lactobacillus buchneri has a neuroprotective effects on SH-SY5Y-cultured neuroblastoma cells (Cheon et al., 2020). The mechanisms by which gut microbiota can influence brain neurochemistry and behaviour are not known, but the participation of the vagus nerve, the production of neuroactive microbial metabolites such as short chain fatty acids (SCFAs) or even bacterial-derived NTs have been proposed (Bauer et al., 2019; Bienenstock et al., 2015; Dinan and Cryan, 2017; Villageliú and Lyte, 2018; Y1lmaz and Gökmen, 2020). Another possibility is the modification by intestinal bacteria-derived metabolites of gut hormone secretion, including glucagon-like peptide1 (GLP1) and peptide YY, leptin and ghrelin which could impact on hypothalamic neuroendocrine pathways to modify appetite (Ejtahed and Hasani-Ranjbar, 2019) and the activation state of several brain areas as the PFC or the HPT (Zanchi et al., 2017). Another intriguing potential mechanism is the production by microorganisms of proteins that have amino acid sequences identical to appetite-regulating peptides including $\alpha-M S H$ and NPY (Fetissov, 2019; Holzer and Farzi, 2014). Omega-3 fatty acids have been also shown to contribute to the brain integrity protection (Haast and Kiliaan, 2015).

A close inspection of the heatmaps indicates that, in the HPT, correlations between productive data and neurotransmitters in $\mathrm{T} 3$ and $\mathrm{T} 4$ are very similar to $\mathrm{T} 1$ and different to $\mathrm{T} 2$. This pattern will reinforce the idea that addition of vegetal protein and B. breve CECT8242 to a HFD reverses the effects of fat. Addition of long chain polyunsaturated omega- 3 fatty acids even introduces negative correlations between productive data and NTs. In the ventral HC, changes introduced by probiotics and omega-3 fatty acids in relationship to HFD are small and, in fact, patterns in $\mathrm{T} 3$ and $\mathrm{T} 4$ are more similar to $\mathrm{T} 2$ than to $\mathrm{T} 1$.

In conclusion, HFD provoked changes in appetite-related neuropeptides and NTs mostly of the dopaminergic pathway when fed to pigs, in a similar way to that described in rodents. These findings confirm the ability of an obesity-inducing diet to provoke changes in the signalling pathways of the brain controlling appetite and other behaviours. Furthermore, they reinforce the validity of the porcine model for nutritional studies.

On the other hand, the inclusion of vegetal protein and B. breve CECT8242, especially if combined with long chain polyunsaturated omega-3 fatty acids as SDA and HDA, is able to partially reverse the changes induced by HFD. Besides the effects of probiotic and unsaturated fatty acid supplementation against weight gain and obesity, their potential benefits on brain function and neurochemical pathways deserves further research.

Our results support the existence of a bottom-up control of brain processes by the gut microbiota and the gut endocrine signalling pathways. The analysis of the microbiota alterations induced by HFD in the porcine model and whether they can be restored by dietary supplementation with $B$. breve and long chain polyunsaturated omega-3 fatty acids, and the elucidation of the physiological mechanisms through which these effects are achieved will be an important step ahead in this important area of research. 


\section{Acknowledgments}

We recognize the efforts of the IRTA technicians Joaquim Soler, Neus Torrentó, Albert Brun, Marina Gispert and Agustí Quintana for the animal care and their participation in the process of data and sample collection.

\section{Funding details}

This work was supported by CDTI (Centro para el Desarrollo Tecnológico e Industrial, Spain) and Programa Operativo FEDER, Project references: IPT-20111008 (Acronym INCOMES) and IDI-20141206 (Acronym SMARTFOODS).

\section{Disclosure statement}

José A. Moreno-Muñoz and María Rodriguez-Palmero are employees of the company Laboratorios Ordesa, S.L.

\section{References}

Arora, T., Singh, S., Sharma, R.K., 2013. Probiotics: Interaction with gut microbiome and antiobesity potential. Nutrition 29, 591-596. https://doi.org/10.1016/j.nut.2012.07.017

Arroyo, L., Carreras, R., Valent, D., Peña, R., Mainau, E., Velarde, A., Sabrià, J., Bassols, A., 2016. Effect of handling on neurotransmitter profile in pig brain according to fear related behaviour. Physiol. Behav. 167, 374-381. https://doi.org/10.1016/j.physbeh.2016.10.005

Bagarolli, R.A., Tobar, N., Oliveira, A.G., Araújo, T.G., Carvalho, B.M., Rocha, G.Z., Vecina, J.F., Calisto, K., Guadagnini, D., Prada, P.O., Santos, A., Saad, S.T.O., Saad, M.J.A., 2017. Probiotics modulate gut microbiota and improve insulin sensitivity in DIO mice. J. Nutr. Biochem. 50, 16-25. https://doi.org/10.1016/j.jnutbio.2017.08.006

Bassols, A., Costa, C., Eckersall, P.D., Osada, J., Sabrià, J., Tibau, J., 2014. The pig as an animal model for human pathologies: a proteomics perspective. Proteomics. Clin. Appl. 8, 715-731. https://doi.org/10.1002/prca.201300099

Bauer, K.C., Rees, T., Finlay, B.B., 2019. The Gut Microbiota-Brain Axis Expands Neurologic Function: A Nervous Rapport. Bioessays 41, e1800268. https://doi.org/10.1002/bies.201800268

Beck, B., Pourié, G., 2013. Ghrelin, neuropeptide Y, and other feeding-regulatory peptides active in the hippocampus: role in learning and memory. Nutr. Rev. 71, 541-561. https://doi.org/10.1111/nure.12045

Beilharz, J., Maniam, J., Morris, M., 2015. Diet-Induced Cognitive Deficits: The Role of Fat and Sugar, Potential Mechanisms and Nutritional Interventions. Nutrients 7, 6719-6738. https://doi.org/10.3390/nu7085307

Bermúdez-Humarán, L.G., Salinas, E., Ortiz, G.G., Ramirez-Jirano, L.J., Morales, J.A., Bitzer-Quintero, O.K., 2019. From Probiotics to Psychobiotics: Live Beneficial Bacteria Which Act on the Brain-Gut Axis. Nutrients 11. https://doi.org/10.3390/nu11040890

Bernini, L.J., Simão, A.N.C., Alfieri, D.F., Lozovoy, M.A.B., Mari, N.L., de Souza, C.H.B., Dichi, I., Costa, G.N., 2016. Beneficial effects of Bifidobacterium lactis on lipid profile and cytokines in patients with metabolic syndrome: A randomized trial. Effects of probiotics on metabolic syndrome. Nutrition 32, 716-719. https://doi.org/10.1016/j.nut.2015.11.001 
Bienenstock, J., Kunze, W., Forsythe, P., 2015. Microbiota and the gut-brain axis. Nutr. Rev. 73, 28-31. https://doi.org/10.1093/nutrit/nuv019

Cano, P.G., Santacruz, A., Trejo, F.M., Sanz, Y., 2013. Bifidobacterium CECT 7765 improves metabolic and immunological alterations associated with obesity in high-fat diet-fed mice. Obesity 21, 2310-2321. https://doi.org/10.1002/oby.20330

Cao, G., Tao, F., Hu, Y., Li, Z., Zhang, Y., Deng, B., Zhan, X., 2019. Positive effects of a: Clostridium butyricum-based compound probiotic on growth performance, immune responses, intestinal morphology, hypothalamic neurotransmitters, and colonic microbiota in weaned piglets. Food Funct. 10, 2926-2934. https://doi.org/10.1039/c8fo02370k

Chen, J., Wang, R., Li, X.-F., Wang, R.-L., 2012. Bifidobacterium adolescentis supplementation ameliorates visceral fat accumulation and insulin sensitivity in an experimental model of the metabolic syndrome. Br. J. Nutr. 107, 1429-1434. https://doi.org/10.1017/S0007114511004491

Cheon, Lim, Lee, Paik, 2020. Probiotic Properties and Neuroprotective Effects of Lactobacillus buchneri KU200793 Isolated from Korean Fermented Foods. Int. J. Mol. Sci. 21, 1227. https://doi.org/10.3390/ijms21041227

Dinan, T.G., Cryan, J.F., 2017. The Microbiome-Gut-Brain Axis in Health and Disease. Gastroenterol. Clin. North Am. https://doi.org/10.1016/j.gtc.2016.09.007

Dziedzic, B., Szemraj, J., Bartkowiak, J., Walczewska, A., 2007. Various Dietary Fats Differentially Change the Gene Expression of Neuropeptides Involved in Body Weight Regulation in Rats. J. Neuroendocrinol. 19, 364-373. https://doi.org/10.1111/j.13652826.2007.01541.x

Ejtahed, H.-S., Hasani-Ranjbar, S., 2019. Neuromodulatory effect of microbiome on gut-brain axis; new target for obesity drugs. J. Diabetes Metab. Disord. 18, 263-265. https://doi.org/10.1007/s40200-019-00384-4

Fanselow, M.S., Dong, H.-W., 2010. Are the Dorsal and Ventral Hippocampus Functionally Distinct Structures? Neuron 65, 7-19. https://doi.org/10.1016/j.neuron.2009.11.031

Fernø, J., Señarís, R., Diéguez, C., Tena-Sempere, M., López, M., 2015. Orexins (hypocretins) and energy balance: More than feeding. Mol. Cell. Endocrinol. 418, 17-26. https://doi.org/10.1016/j.mce.2015.07.022

Fetissov, S.O., 2019. Neuropeptide-like signaling in the microbiota-gut-brain axis. Behav. Brain Sci. 42. https://doi.org/10.1017/s0140525x18002765

Galland, L., 2014. The gut microbiome and the brain. J. Med. Food 17, 1261-72. https://doi.org/10.1089/jmf.2014.7000

González-Arancibia, C., Urrutia-Piñones, J., Illanes-González, J., Martinez-Pinto, J., Sotomayor-Zárate, R., Julio-Pieper, M., Bravo, J.A., 2019. Do your gut microbes affect your brain dopamine? Psychopharmacology (Berl). https://doi.org/10.1007/s00213-01905265-5

Gumbs, M.C.R., van den Heuvel, J.K., la Fleur, S.E., 2016. The effect of obesogenic diets on brain Neuropeptide Y. Physiol. Behav. 162, 161-173. https://doi.org/10.1016/j.physbeh.2016.04.049

Haast, R.A.M., Kiliaan, A.J., 2015. Impact of fatty acids on brain circulation, structure and function. Prostaglandins, Leukot. Essent. Fat. Acids 92, 3-14. 
https://doi.org/10.1016/j.plefa.2014.01.002

Heinritz, S.N., Mosenthin, R., Weiss, E., 2013. Use of pigs as a potential model for research into dietary modulation of the human gut microbiota. Nutr. Res. Rev. 26, 191-209. https://doi.org/10.1017/S0954422413000152

Holzer, P., Farzi, A., 2014. Neuropeptides and the microbiota- Gut-brain axis. Adv. Exp. Med. Biol. 817, 196-219. https://doi.org/10.1007/978-1-4939-0897-4_9

Hommel, J.D., Trinko, R., Sears, R.M., Georgescu, D., Liu, Z.W., Gao, X.B., Thurmon, J.J., Marinelli, M., DiLeone, R.J., 2006. Leptin Receptor Signaling in Midbrain Dopamine Neurons Regulates Feeding. Neuron 51, 801-810. https://doi.org/10.1016/j.neuron.2006.08.023

Huang, C.-W., Chien, Y.-S., Chen, Y.-J., Ajuwon, K.M., Mersmann, H.M., Ding, S.-T., 2016. Role of n-3 Polyunsaturated Fatty Acids in Ameliorating the Obesity-Induced Metabolic Syndrome in Animal Models and Humans. Int. J. Mol. Sci. 17, 1689. https://doi.org/10.3390/ijms17101689

Kang, J.X., Gleason, E.D., 2013. Omega-3 Fatty acids and hippocampal neurogenesis in depression. CNS Neurol. Disord. Drug Targets 12, 460-5.

Kondo, S., Kamei, A., Xiao, J.Z., Iwatsuki, K., Abe, K., 2013. Bifidobacterium breve B-3 exerts metabolic syndrome-suppressing effects in the liver of diet-induced obese mice: a DNA microarray analysis. Benef. Microbes 4, 247-251. https://doi.org/10.3920/BM2012.0019

Kondo, S., Xiao, J., Satoh, T., Odamaki, T., Takahashi, S., Sugahara, H., Yaeshima, T., Iwatsuki, K., Kamei, A., Abe, K., 2010. Antiobesity Effects of Bifidobacterium breve Strain B-3 Supplementation in a Mouse Model with High-Fat Diet-Induced Obesity. Biosci. Biotechnol. Biochem. 74, 1656-1661. https://doi.org/10.1271/bbb.100267

Koopmans, S.J., Schuurman, T., 2015. Considerations on pig models for appetite, metabolic syndrome and obese type 2 diabetes: From food intake to metabolic disease. Eur. J. Pharmacol. 759, 231-239. https://doi.org/10.1016/j.ejphar.2015.03.044

Krishna, S., Keralapurath, M.M., Lin, Z., Wagner, J.J., de La Serre, C.B., Harn, D.A., Filipov, N.M., 2015. Neurochemical and electrophysiological deficits in the ventral hippocampus and selective behavioral alterations caused by high-fat diet in female C57BL/6 mice. Neuroscience 297, 170-181. https://doi.org/10.1016/j.neuroscience.2015.03.068

Lam, D.D., Garfield, A.S., Marston, O.J., Shaw, J., Heisler, L.K., 2010. Brain serotonin system in the coordination of food intake and body weight. Pharmacol. Biochem. Behav. 97, 84-91. https://doi.org/10.1016/j.pbb.2010.09.003

Lim, S.-M., Kim, D.-H., 2017. Bifidobacterium adolescentis IM38 ameliorates high-fat dietinduced colitis in mice by inhibiting NF- $\mathrm{KB}$ activation and lipopolysaccharide production by gut microbiota. Nutr. Res. 41, 86-96. https://doi.org/10.1016/j.nutres.2017.04.003

Liu, Y.-W., Liu, W.-H., Wu, C.-C., Juan, Y.-C., Wu, Y.-C., Tsai, H.-P., Wang, S., Tsai, Y.C., 2016. Psychotropic effects of Lactobacillus plantarum PS128 in early life-stressed and naïve adult mice. Brain Res. 1631, 1-12. https://doi.org/10.1016/j.brainres.2015.11.018

Lorente-Cebrián, S., Costa, A.G. V., Navas-Carretero, S., Zabala, M., Martínez, J.A., Moreno-Aliaga, M.J., 2013. Role of omega-3 fatty acids in obesity, metabolic syndrome, and cardiovascular diseases: a review of the evidence. J. Physiol. Biochem. 69, 633-651. https://doi.org/10.1007/s13105-013-0265-4 
Mantzoros, C.S., Magkos, F., Brinkoetter, M., Sienkiewicz, E., Dardeno, T.A., Kim, S., Hamnvik, O.R., Koniaris, A., 2011. Leptin in human physiology and pathophysiology. AJP Endocrinol Metab 301, 567-584. https://doi.org/10.1152/ajpendo.00315.2011.

Martínez-Fernández, L., Laiglesia, L.M., Huerta, A.E., Martínez, J.A., Moreno-Aliaga, M.J., 2015. Omega-3 fatty acids and adipose tissue function in obesity and metabolic syndrome. Prostaglandins Other Lipid Mediat. 121, 24-41. https://doi.org/10.1016/j.prostaglandins.2015.07.003

Mauricio, M.D., Serna, E., Fernández-Murga, M.L., Portero, J., Aldasoro, M., Valles, S.L., Sanz, Y., Vila, J.M., 2017. Bifidobacterium pseudocatenulatum CECT 7765 supplementation restores altered vascular function in an experimental model of obese mice. Int. J. Med. Sci. 14, 444-451. https://doi.org/10.7150/ijms.18354

Mayer, E.A., Tillisch, K., Gupta, A., 2015. Gut/brain axis and the microbiota. J. Clin. Invest. 125, 926-38. https://doi.org/10.1172/JCI76304

Minami, J., Kondo, S., Yanagisawa, N., Odamaki, T., Xiao, J., Abe, F., Nakajima, S., Hamamoto, Y., Saitoh, S., Shimoda, T., 2015. Oral administration of Bifidobacterium breve B-3 modifies metabolic functions in adults with obese tendencies in a randomised controlled trial. J. Nutr. Sci. 4, e17. https://doi.org/10.1017/jns.2015.5

Morris, M.J., Chen, H., Watts, R., Shulkes, A., Cameron-Smith, D., 2008. Brain neuropeptide $\mathrm{Y}$ and CCK and peripheral adipokine receptors: temporal response in obesity induced by palatable diet. Int. J. Obes. 32, 249-258. https://doi.org/10.1038/sj.ijo.0803716

Moya-Pérez, A., Romo-Vaquero, M., Tomás-Barberán, F., Sanz, Y., García-Conesa, M.-T., 2014. Hepatic molecular responses to Bifidobacterium pseudocatenulatum CECT 7765 in a mouse model of diet-induced obesity. Nutr. Metab. Cardiovasc. Dis. 24, 57-64. https://doi.org/10.1016/j.numecd.2013.04.011

Noble, E.E., Hsu, T.M., Kanoski, S.E., 2017. Gut to Brain Dysbiosis: Mechanisms Linking Western Diet Consumption, the Microbiome, and Cognitive Impairment. Front. Behav. Neurosci. 11, 9. https://doi.org/10.3389/fnbeh.2017.00009

Nobunaga, M., Obukuro, K., Kurauchi, Y., Hisatsune, A., Seki, T., Tsutsui, M., Katsuki, H., 2014. High fat diet induces specific pathological changes in hypothalamic orexin neurons in mice. Neurochem. Int. 78, 61-66. https://doi.org/10.1016/j.neuint.2014.09.002

Nova, E., Pérez de Heredia, F., Gómez-Martínez, S., Marcos, A., 2016. The Role of Probiotics on the Microbiota. Nutr. Clin. Pract. 31, 387-400. https://doi.org/10.1177/0884533615620350

Palmiter, R.D., 2007. Is dopamine a physiologically relevant mediator of feeding behavior? Trends Neurosci. 30, 375-381. https://doi.org/10.1016/j.tins.2007.06.004

Park, E.S., Yi, S.J., Kim, J.S., Lee, H.S., Lee, I.S., Seong, J.K., Jin, H.K., Yoon, Y.S., 2004. Changes in orexin-A and neuropeptide $Y$ expression in the hypothalamus of the fasted and high-fat diet fed rats. J. Vet. Sci. 5, 295-302.

Reichold, A., Brenner, S.A., Spruss, A., Förster-Fromme, K., Bergheim, I., Bischoff, S.C., 2014. Bifidobacterium adolescentis protects from the development of nonalcoholic steatohepatitis in a mouse model. J. Nutr. Biochem. 25, 118-125. https://doi.org/10.1016/j.jnutbio.2013.09.011

Rezaeiasl, Z., Salami, M., Sepehri, G., 2019. The effects of probiotic Lactobacillus and Bifidobacterium strains on memory and learning behavior, long-term potentiation (LTP), 
and some biochemical parameters in $\beta$-amyloid-induced rat's model of Alzheimer's disease. Prev. Nutr. Food Sci. 24, 265-273. https://doi.org/10.3746/pnf.2019.24.3.265

Rhee, S.H., Pothoulakis, C., Mayer, E.A., 2009. Principles and clinical implications of the brain-gut-enteric microbiota axis. Nat. Rev. Gastroenterol. Hepatol. https://doi.org/10.1038/nrgastro.2009.35

Savignac, H.M., Couch, Y., Stratford, M., Bannerman, D.M., Tzortzis, G., Anthony, D.C., Burnet, P.W.J., 2016. Prebiotic administration normalizes lipopolysaccharide (LPS)induced anxiety and cortical 5-HT2A receptor and IL1- $\beta$ levels in male mice. Brain. Behav. Immun. 52, 120-131. https://doi.org/10.1016/j.bbi.2015.10.007

Savignac, H.M., Kiely, B., Dinan, T.G., Cryan, J.F., 2014. Bifidobacteria exert strain-specific effects on stress-related behavior and physiology in BALB/c mice. Neurogastroenterol. Motil. 26, 1615-1627. https://doi.org/10.1111/nmo.12427

Savignac, H.M., Tramullas, M., Kiely, B., Dinan, T.G., Cryan, J.F., 2015. Bifidobacteria modulate cognitive processes in an anxious mouse strain. Behav. Brain Res. 287, 59-72. https://doi.org/10.1016/j.bbr.2015.02.044

Schwartz, M.W., Woods, S.C., Porte, D., Seeley, R.J., Baskin, D.G., 2000. Central nervous system control of food intake. Nature 404, 661-671. https://doi.org/10.1038/35007534

Serrano, J., Cassañé, A., Font-Fornols, M., Puigjaner Riba, J., Moreno, J., RodriguezPalmero, M Jiménez, J., Quintanilla, R., Arola, L., Portero-Otin, M., Tibau, J., 2016. Rice protein hydrolysate and probiotic bifidobacterium breve combined with omega-3 fatty acids prevent weight gain and associated metabolic changes in a porcine model of prepuberal obesity, in: Journal of Pediatric Gastroenterology and Nutrition. p. N-eP-024.

Shin, J.-H., Nam, M.H., Lee, H., Lee, J.-S., Kim, H., Chung, M.-J., Seo, J.-G., 2017. Amelioration of obesity-related characteristics by a probiotic formulation in a high-fat diet-induced obese rat model. Eur. J. Nutr. https://doi.org/10.1007/s00394-017-1481-4

Sobrino Crespo, C., Perianes Cachero, A., Puebla Jiménez, L., Barrios, V., Arilla Ferreiro, E., 2014. Peptides and food intake. Front. Endocrinol. (Lausanne). 5, 58. https://doi.org/10.3389/fendo.2014.00058

Stevens, G.A., Singh, G.M., Lu, Y., Danaei, G., Lin, J.K., Finucane, M.M., Bahalim, A.N., McIntire, R.K., Gutierrez, H.R., Cowan, M., Paciorek, C.J., Farzadfar, F., Riley, L., Ezzati, M., 2012. National, regional, and global trends in adult overweight and obesity prevalences. Popul. Health Metr. 10, 22. https://doi.org/10.1186/1478-7954-10-22

Stricker-Krongrad, A., Cumin, F., Burlet, C., Beck, B., 1998. Hypothalamic neuropeptide Y and plasma leptin after long-term high-fat feeding in the rat. Neurosci. Lett. 254, 157-60.

van de Wouw, M., Schellekens, H., Dinan, T.G., Cryan, J.F., 2017. Microbiota-Gut-Brain Axis: Modulator of Host Metabolism and Appetite. J. Nutr. 147, 727-745. https://doi.org/10.3945/jn.116.240481

van Zessen, R., van der Plasse, G., Adan, R. a. H., 2012. Contribution of the mesolimbic dopamine system in mediating the effects of leptin and ghrelin on feeding. Proc. Nutr. Soc. 71, 435-445. https://doi.org/10.1017/S0029665112000614

Villageliú, D., Lyte, M., 2018. Dopamine production in Enterococcus faecium: A microbial endocrinology-based mechanism for the selection of probiotics based on neurochemicalproducing potential. PLoS One 13, e0207038. https://doi.org/10.1371/journal.pone.0207038 
Wang, H., Storlien, L.H., Huang, X.-F., 2002. Effects of dietary fat types on body fatness, leptin, and ARC leptin receptor, NPY, and AgRP mRNA expression. Am. J. Physiol. Metab. 282, E1352-E1359. https://doi.org/10.1152/ajpendo.00230.2001

Weltens, N., Iven, J., Van Oudenhove, L., Kano, M., 2018. The gut-brain axis in health neuroscience: implications for functional gastrointestinal disorders and appetite regulation. Ann. N. Y. Acad. Sci. https://doi.org/10.1111/nyas.13969

Westfall, S., Lomis, N., Kahouli, I., Dia, S.Y., Singh, S.P., Prakash, S., 2017. Microbiome, probiotics and neurodegenerative diseases: deciphering the gut brain axis. Cell. Mol. Life Sci. https://doi.org/10.1007/s00018-017-2550-9

Y1lmaz, C., Gökmen, V., 2020. Neuroactive compounds in foods: Occurrence, mechanism and potential health effects. Food Res. Int. 128, 108744. https://doi.org/10.1016/j.foodres.2019.108744

Yong, S.J., Tong, T., Chew, J., Lim, W.L., 2020. Antidepressive Mechanisms of Probiotics and Their Therapeutic Potential. Front. Neurosci. https://doi.org/10.3389/fnins.2019.01361

Yoo, S.-R., Kim, Y.-J., Park, D.-Y., Jung, U.-J., Jeon, S.-M., Ahn, Y.-T., Huh, C.-S., McGregor, R., Choi, M.S., 2013. Probiotics L. plantarum and L. curvatus in Combination Alter Hepatic Lipid Metabolism and Suppress Diet-Induced Obesity. Obesity 21, 2571-2578. https://doi.org/10.1002/oby.20428

Yu, J.H., Kim, M.-S., 2012. Molecular Mechanisms of Appetite Regulation. Diabetes Metab J 36, 391-398. https://doi.org/10.4093/dmj.2012.36.6.391

Zanchi, D., Depoorter, A., Egloff, L., Haller, S., Mählmann, L., Lang, U.E., Drewe, J., Beglinger, C., Schmidt, A., Borgwardt, S., 2017. The impact of gut hormones on the neural circuit of appetite and satiety: A systematic review. Neurosci. Biobehav. Rev. https://doi.org/10.1016/j.neubiorev.2017.06.013

Ziotopoulou, M., Mantzoros, C.S., Hileman, S.M., Flier, J.S., 2000. Differential expression of hypothalamic neuropeptides in the early phase of diet-induced obesity in mice. Am. J. Physiol. Metab. 279, E838-E845. https://doi.org/10.1152/ajpendo.2000.279.4.E838 